\title{
THE FLUORESCENCE AND ABSORPTION OF ANTHRACENE.
}

By Louise Sherwood MCDowell.

\begin{abstract}
A NHRACENE, because of its intense blue-violet fluorescence, A offers a promising opportunity for the comparative study of the fluorescence of a substance in its different forms : solid, liquid, and solution. The present study was undertaken, in order to make such a comparison, at the suggestion of Professors Nichols and Merritt, of whose inspiring interest the author wishes to express her grateful appreciation. Owing to the impurity of the anthracene used, the work naturally fell into three divisions : (I) the study of the fluorescence of impure anthracene; (2) the purification of the anthracene; (3) the study of the fluorescence of pure anthracene.
\end{abstract}

\section{Nature of Anthracene.}

Anthracene is a benzene derivative, prepared from those portions of coal tar which boil between $340^{\circ}$ and $360^{\circ} \mathrm{C}$. In its pure form the anthracene crystallizes in monoclinic plates, and has a blueviolet fluorescence. It melts at $2 \mathrm{I}^{\circ} \mathrm{C}$. and boils at $35 \mathrm{I}^{\circ} \mathrm{C}$. The crude anthracene contains an impurity of unknown composition, chrysogen, the slightest trace of which has been supposed entirely to destroy the blue-violet fluorescence.

\section{History.}

No study of the fluorescence of the crude anthracene has, so far as the writer is aware, yet been undertaken. The study of the fluorescence of the pure anthracene has been chiefly confined to the solutions. In this field, the work of two men needs to be mentioned, J. Hartley, in England, and O. Knoblauch, in Germany. Hartley ${ }^{1}$ studied the absorption bands of solutions of pure anthracene in glacial acetic acid diluted with absolute alcohol. The solutions varied in concentration from I part of anthracene in 1,000 of the 
solvent to I part in 50,000,000. He found four absorption bands in the following positions : $\lambda .3600-.3607 \mu ; .3430-.3434 \mu ; .3285-$ $.3287 \mu$. The least refrangible was not measurable on his scale.

O. Knoblauch, ${ }^{1}$ in a general study of the intensity of fluorescence of various solutions, studied ten solutions of pure anthracene, which he arranged in order of intensity of fluorescence, as follows : benzol $=$ toluol $=$ xylol $=$ isobutyl alcohol $>$ amyl alcohol $=$ ethyl alcohol $=$ ether $>$ acetone $>$ petroleum ether $>$ chloroform.

In this country E. L. Nichols and E. Merritt, ${ }^{2}$ in a study of the fluorescence and phosphorescence of various organic substances at low temperatures, have found that, at the temperature of liquid air, the solid still shows the characteristic blue-violet fluorescence unchanged in intensity, and also a white phosphorescence.

Within the past year, T. S. Elston ${ }^{3}$ has made a study of the fluorescence of the vapor of pure anthracene and found that the fluorescence spectrum extends from $\lambda .365$ to $.470 \mu$, with bright bands at $\lambda .390, .4 \mathrm{I} 5$, and $.432 \mu$, and that the absorption spectrum extends continuously from about $\lambda .400 \mu$ to some point beyond $\lambda .325 \mu$.

\section{Fluorescence of Impure Anthracene.}

The present experiments were begun with a partially purified, crystallized anthracene, yellow in color. This showed a bright green fluorescence. To put in convenient form for photographing, the anthracene was melted between two microscope slides, and allowed to cool. The layer so formed was about a millimeter in thickness. In this form the anthracene showed three distinct absorption bands, the position of which was found photographically. The plate was placed close to the tube of the spectrograph; the carbon arc light focused upon it; and an exposure of ten to twelve seconds made. Of the five exposures on each plate, the first and fifth were of the potassium and strontium arcs respectively. By means of these two reference spectra, a calibration curve was plotted, and the position of the absorption bands read from the curve. See plate at close of article. The bands were found to have the following positions :

\footnotetext{
${ }^{1}$ Knoblauch, O., Wiedemann's Annalen, 54, 192. ${ }^{2}$ Nichols and Merritt, Physical Review, 18, 355.

${ }^{3}$ T. S. Elston, Astrophysical Journal, April, I907.
} 
No. 2.] FLUORESCENCE AND ABSORPTION OF ANTHR ACENE. I 57

$\begin{array}{cc}\text { Extent. } & \text { Middle Point. } \\ \lambda & \lambda \\ 4,990-4,870 & 4,930 \\ 4,620-4,520 & 4,570\end{array}$

For the fluorescence, the mercury arc lamp of the Arons Lummer type was used as an exciting light, and also as a reference spectrum. The first four exposures on each plate were of the fluorescence spectrum, for which the apparatus was so arranged that the spectrograph and lamp were at right angles and the plate placed with its

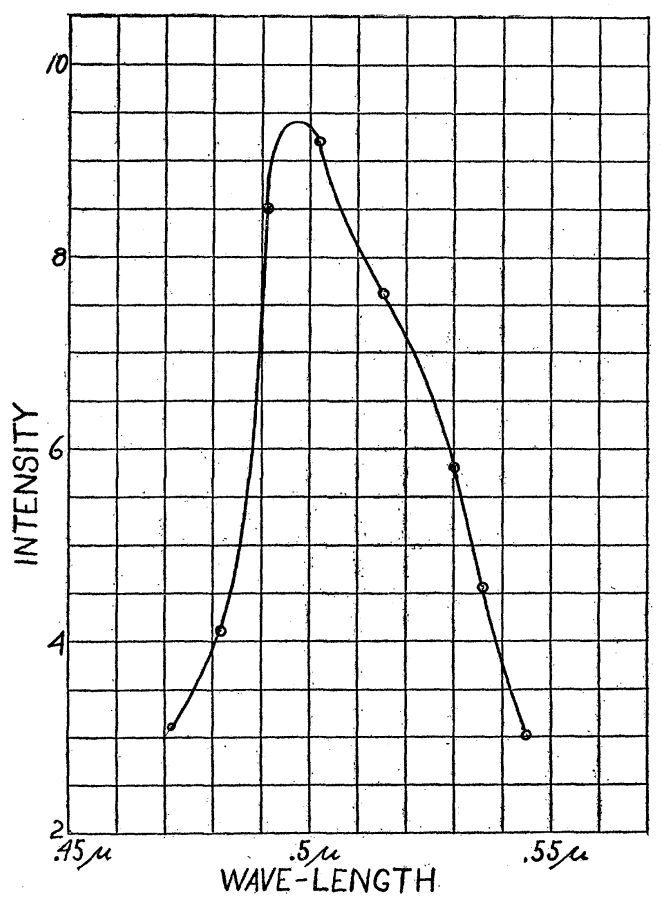

edge vertical and directly in front of the slit of the spectrograph. For the fifth exposure, the light of the mercury arc was reflected directly into the spectrograph by a glass mirror placed at an angle of $45^{\circ}$. The time of exposure varied, according to the width of slit, from five minutes to an hour. In order to test the effect of the glass, one photograph was taken in which the plate was replaced by a solid cylinder of anthracene about $.8 \mathrm{~cm}$. in diameter; and the 
[Vol. XXVI.

light from the mercury arc was passed through a violet glass to cut off the green light. The resulting photographs show two fluorescence bands, of which the first is but faintly visible in the photograph, although it appears to the eye the more intense. See plate at close of article. The position of the bands is :

Band I. $\quad \stackrel{\lambda}{\lambda}, 440-5,250$

Band II. 5, 145-4,865

A photograph of the fluorescence of solid methyl anthracene taken in the same way showed these two green bands, and in addition a faint blue fluorescence extending to line $.4358 \mu$ of the mercury spectrum.

As a check upon the results obtained photographically, the intensity curve for the green fluorescence bands was taken by means of a Lummer-Brodhun spectrophotometer. The iron spark was used as an exciting light. In order to avoid stray light, the visible portion of the spectrum, formed through a quartz lens and prism, was. intercepted by a screen, and only the ultra-violet rays allowed to fall upon the plate of anthracene. In this case the plate was formed by subliming the anthracene on glass. As a comparison light there was used an acetylene flame reflected at an angle of about $45^{\circ}$ from a block of magnesium carbonate. To prevent light from this source falling on the plate the acetylene flame, except for the circular opening through which light fell upon the reflecting surface, was completely enclosed by a black screen. The results are shown in the accompanying curve. To test the killing effect of the extreme red and infra-red rays upon the green fluorescence, the light of the arc lantern was intercepted by a thin rubber screen, and the rays which passed through allowed to fall at grazing incidence upon the fluorescing surface. The intensity of the fluorescence as observed through the spectrophotometer remained unchanged.

For the determination of the rays which excite the green fluorescence of the solid, the spectra of the carbon arc lamp, the mercury arc, the Nernst glower, and the iron spark, were successively thrown on a screen, and the plate of sublimed anthracene moved slowly through the spectrum. In the last case, the spectrum was produced by a quartz lens and prism, to avoid the absorption 
No. 2.] FLUORESCENCE AND ABSORPTION OF ANTHRACENE. I 59

due to glass. The results show that, with the possible exception of two narrow bands in the blue, all rays are effective from the green to the extreme ultra-violet.

The study of the fluorescence of the liquid anthracene involved several difficulties. The anthracene, if melted too rapidly, decomposes ; also it begins to sublime at temperatures far below the melting point, and if removed from the heat, coats over the surface before an observation can be taken. To obviate these difficulties, the anthracene was placed in a long glass tube about one and one half centimeters in diameter, and placed in an electric heater, $6 \times 6 \times \mathrm{I}$ in. The whole was covered with asbestos paper, except for openings through which the tube and thermometer were received, the light admitted, and observations taken. For the absorption bands, both a gas flame and a focused carbon arc were used, but no absorption bands were visible through the spectroscope. Throughout the experiment, the thermometer was kept only a few degrees above the melting point, yet the solution under the influence of the heat rapidly decomposed, turned orange, and then dark brown until it became almost opaque to light. For the fluorescence the plan was first tried of sealing the metal terminals for a spark into the glass tube, but the anthracene vapor attacked the terminals and caused them to burn out so rapidly that the plan had to be abandoned. Thereafter the spark was placed just outside the glass tube. Under these conditions, the liquid showed no fluorescence when excited by the spark between terminals of aluminum, zinc, tin, or iron, by the mercury vapor lamp, or the carbon arc.

To test the fluorescence of impure anthracene in solution, the following solvents were used : benzol, toluol, xylol, isobutyl alcohol, amyl alcohol, ethyl alcohol (absolute), ether, acetone, petroleum ether, chloroform, carbon disulphide, and glacial acetic acid. Concentrated solutions were first made and then diluted in the ratios $\mathrm{I}: 2, \mathrm{I}: 4, \mathrm{I}: 8, \mathrm{I}: \mathrm{I} 6, \mathrm{I}: 32$. In ether and acetone solutions, through cells three centimeters thick, there were visible very faint absorption bands, too faint to affect the photographic plate when exposures for 25 minutes were made. In this case the light was that of the ordinary gas burner. No fluorescence was observable under any of the excitations previously tried. In testing the exciting powers 
of the ultra-violet rays small rectangular glass cells $5.65 \times 3 \times \mathrm{I}$ $\mathrm{cm}$. (inside measurement) were nearly filled with the solution to be tested ; the open top covered by a quartz plate ; and the metal sparks of aluminum, zinc, tin, iron, and copper successively placed above. In no case was fluorescence visible.

\section{Purification of Anthracene.}

In the course of the determination of the exciting rays, an attempt was made to use plates prepared by evaporating a chloroform solution on glass. When a plate so prepared was held in the ultraviolet spectrum of the iron spark it fluoresced, but with a faint blue color, suggestive of pure anthracene. A similar test of other solutions, which had been standing in a dimly lighted room for several days, gave the following results :

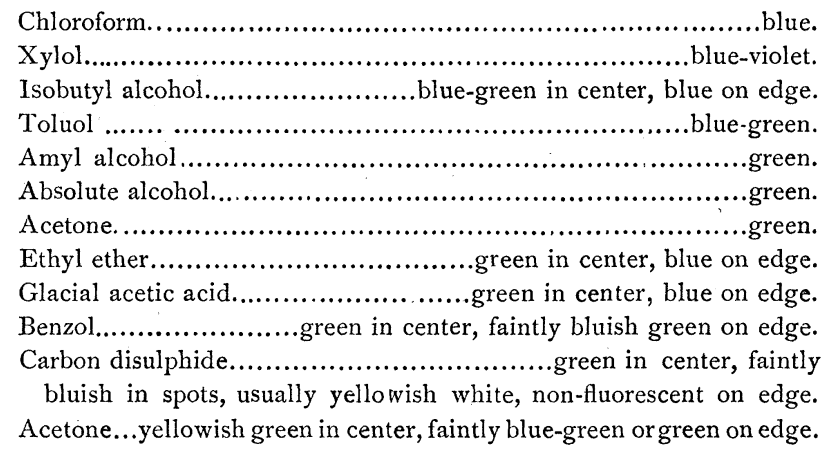

Obviously the next step was to decide the relative effects of solvent, concentration, light, and time, in producing the change. For this purpose fresh solutions, saturated and dilute, were made in the most promising solvents : chloroform, xylol, and isobutyl alcohol. Portions of these solutions were left in diffuse daylight and in a photographic dark room. Each day for a week, tests were made by evaporating small portions on glass and placing the evaporated product in the light from the carbon arc, and also from the iron spark. The tests showed :

I. That daylight assists the change although bright sunlight is not essential.

II. That solvents differ in their ability to produce the change, i. e., xylol is better than chloroform. 
NO. 2.] FLUORESCENCE AND ABSORPTION OF ANTHRACENE. I6I

III. That a difference in concentration has little effect upon the change.

IV. That the time required depends upon the brightness of the light and the solvent used.

V. That in some cases the evaporated product, if exposed to air, decomposes.

These results led to a study of the method of Fritzsche, ${ }^{1}$ who was probably the first to undertake the purification of anthracene. His method in brief was this: The crude anthracene was dissolved with heat in a mixture of coal-tar oils (Steinkohlenoel) and placed in the sunlight. The substance which first crystallized out was dissolved in the same way until crystals of the desired purity were obtained.

Berthelot's ${ }^{2}$ method is similar. The coal-tar product left in the retort by the distillation of the substances which boil below $350^{\circ}$ is crystallized again from boiling xylol, and each time the part which crystallizes out on cooling, is pressed out. Then it is again crystallized from alcohol, and finally sublimed at the lowest possible temperature.

In the first experiments performed by the writer, the impure anthracene was dissolved in boiling kerosene, and the saturated solution allowed to stand in sunlight until a portion crystallized out. These crystals were redissolved in fresh kerosene, and the process repeated until the product was very nearly pure white. After the last filtration, the anthracene was washed in cold alcohol, in which it is but slightly soluble. To test the effect of the fresh kerosene, the product which first crystallized out was redissolved in the same solution, allowed to crystallize out a second time, and the process repeated until the yellow color disappeared. The precipitate was finally filtered out and washed as before. No difference in the product so obtained was observable. As a third test, the hot solution of anthracene was kept in the sunlight for fifteen minutes, at a temperature which prevented crystallization; then allowed to crystallize; filtered out, and the precipitate washed in alcohol. Since this product when subjected to the tests described below showed

${ }^{1}$ Fritzsche, Zeitschrift für Chemie, r867, p. 290.

${ }^{2}$ Berthelot, Bulletin de la Société Chimique de Paris, 8, 232. 
the greatest purity of all the kerosene treated products, it is evident :

I. That renewal of solvent is unessential.

II. That repeated crystallization is unessential.

The same method was tried with chloroform and xylol. An attempt to substitute arc light for sunlight showed that owing to the lessened intensity of the light a much longer time was required.

To the products thus purified, various tests were applied. They were found to possess a blue-violet fluorescence, and to dissolve readily in any of the solvents used, giving solutions with a blueviolet fluorescence. The xylol and chloroform products, if not carefully washed, gradually lost their fluorescence and turned a yellowish brown. Sublimation of the products in air showed that the chrysogen was still present, for the sublimate gave in one spot blueviolet fluorescence; in another, green. When the sublimation was gentle, that which was first deposited on the cold plate of glass showed blue-violet fluorescence. As the heat increased the later deposit showed green fluorescence, so that, by illuminating the plate from the one side or the other, the blue-violet or the green fluorescence was obtained. By stopping the gentle sublimation at the proper time, a product was obtained which gave only blue-violet fluorescence, while the rapid sublimation of the residue gave a product of which the green fluorescence was more intense than any yet observed. Apparently by this means there was secured a greater concentration of the chrysogen, although a complete separation was not effected.

To test whether the change in color of the fluorescence was due to the heat to which the product was subjected, the blue-violet sublimate was rapidly resublimed and gave still a blue-violet fluorescence, which grew gradually fainter as the amount of the sublimate was lessened by repeated sublimations. To complete the separation of the pure anthracene the kerosene-treated product was sublimed in a partial vacuum. The crystals were placed in the bottom of a cylindrical tube. About two inches above was a wire triangle, upon which rested a small glass plate. The tube was connected to a mercury pump and exhausted as far as possible, then closed and placed in a bath of salt and water maintained approxi- 
mately at $100^{\circ} \mathrm{C}$. The sublimation was allowed to continue for about two hours, then the plate was removed and tested. By this means regular crystals of pure anthracene were obtained, and in the later fractions somewhat irregular fern-like crystals, the form of which was apparently modified by the presence of the chrysogen. Complete separation of the chrysogen was not effected. An attempt to separate the pure anthracene by sublimation, before treatment with the kerosene, proved unsuccessful.

To account for these results one possible explanation suggested itself, an explanation based upon the following known facts :

I. Anthracene, if allowed to stand in solution in the bright sunlight, gradually goes over into dianthracene $\left(\mathrm{C}_{28} \mathrm{H}_{20}\right)$, which melts at $244^{\circ} \mathrm{C}$., but upon melting reverts to anthracene, the melting point of which is $213^{\circ} \mathrm{C}$.

II. Chrysogen is known to go through an apparently similar transformation, since if ordinary white dianthracene, which has a blue fluorescence, is melted, the resulting product is yellow and fluoresces bright green.

May it not be that, when the anthracene is exposed to sunlight, the chrysogen first goes over into dichrysogen so that the product which crystallizes out is a mixture of anthracene and dichrysogen, and that the latter upon sublimation at high temperatures reverts to chrysogen and thus restores the green color? This would explain the greater ease of separation after the product is crystallized out of solution.

The Fluorescence and Absorption of Pure Anthracene.

In the study of the fluorescence of the solid, a comparison was made of the fluorescence of the original yellow anthracene, the bright green product of rapid sublimation, the blue-violet product of gentle sublimation, and the pure anthracene crystals from the sublimation in vacuo. As exciting lights, the mercury arc and the ultra-violet rays of the iron spark spectrum were used. As before, the method was photographic. For the absorption bands, the light of the carbon arc lamp was focused upon the plates as in the case of the impure anthracene. For the iron spark excitation the ultraviolet rays were allowed to fall on the plate, and the visible spectrum intercepted by a screen. 
Tables I. and II. give the results of the comparison. A question mark in a column indicates that owing to the presence of a mercury arc line, the exact position of the band is uncertain. The last two bands are those obtained for the fluorescence of the impure anthracene. These results indicate that the substance is fluorescent in all stages of the purification, and that the blue-violet fluorescence is not entirely killed by the presence of the chrysogen. In the purer

TABLE I.

Fluorescence of Solid Anthracene of Different Degrees of Purity.

\begin{tabular}{|c|c|c|c|c|c|c|c|c|}
\hline \multirow{3}{*}{$\begin{array}{c}\text { Substance. } \\
\text { G.S. } \\
\text { O.S. }\end{array}$} & \multirow{3}{*}{$\begin{array}{l}\text { Time. } \\
\begin{array}{l}30 \mathrm{~min} . \\
30 ،\end{array}\end{array}$} & \multicolumn{5}{|c|}{ Bands. } & \multirow{3}{*}{$\begin{array}{c}\text { Extent. } \\
4,860-5,370 \\
4,130-5,370\end{array}$} & \multirow{3}{*}{$\begin{array}{c}\text { Excitant. } \\
\begin{array}{c}\text { M.A. } \\
\text { ، }\end{array}\end{array}$} \\
\hline & & $?$ & & & 5,010 & 5,310 & & \\
\hline & & 4,358 & & & 5,010 & 5,310 & & \\
\hline B. + G. & $45 ،$ & 4,358 & & & 4,985 & 5,310 & $4,130-5,370$ & "6 \\
\hline V.B. & $90 ،$ & 4,358 & & 4,730 & 4,985 & & & "6 \\
\hline G.S. & $45 ،$ & & & & 4,975 & 5,280 & $4,850-5,375$ & 6 \\
\hline B. $+\mathrm{G}$. & $90 ،$ & 4,358 & & & 4,980 & & $4,140-5,080$ & "6 \\
\hline G.S. & $75 ،$ & 4,250 & 4,510 & 4,750 & 4,960 & & $4,190-5,060$ & U.V. \\
\hline O.S. & $60 ،$ & 4,260 & 4,490 & 4,750 & 4,980 & & $4,190-5,090$ & ، \\
\hline V.B. & 120 ، & 4,260 & 4,490 & & 4,960 & & $4,190-5,030$ & 6 \\
\hline B. + G. & $60 ،$ & 4,260 & 4,510 & & 4,960 & & $4,190-5,060$ & 6 \\
\hline P.A. & 120 & 4,220 & 4,470 & & & & $4,100-4,750$ & "6 \\
\hline 0 & 120 ، & & & & 4,940 & & $4,870-5,010$ & "، \\
\hline
\end{tabular}

TABLE II.

Position of Absorption Bands of Solid Anthracene.

\begin{tabular}{|c|c|c|c|c|}
\hline \multirow{3}{*}{$\begin{array}{c}\text { Substance. } \\
\text { O.S. } \\
\text { G.S. }\end{array}$} & \multirow{2}{*}{$\frac{\text { Time. }}{12 \mathrm{sec} .}$} & \multicolumn{3}{|c|}{ Width of Bands. } \\
\hline & & 4,090 & $4,840-4,900$ & $4,540-4,615$ \\
\hline & 20 & 4,090 & $4,860-4,925$ & $4,570-4,650$ \\
\hline B. + G. & 20 & 4,090 & & \\
\hline V.B. & 256 & 4,100 & & \\
\hline V.B. & 25 & 4,100 & & \\
\hline
\end{tabular}

G.S. = green sublimate.

O.S. = original anthracene sublimed.

B. $+\mathrm{G}$. = blue and green sublimate.

P.A. $=$ crystals of pure anthracene.

O. = original anthracene melted on glass.

$\mathrm{V} . \mathrm{B} .=$ violet-blue sublimate.

M.A. = mercury arc.

U.V. = ultra-violet rays of iron spark.

Arc $=$ carbon arc through glass. 
No. 2.] FLUORESCENCE AND ABSORPTION OF ANTHRACENE. I65

specimens, however, the violet fluorescence was relatively strong, the green weak, and in the most impure products, the violet was so faint as to be barely visible. See plate.

For the study of the absorption and fluorescence of the solutions of pure anthracene, the kerosene-treated product was used. Small amounts of concentrated solutions in the following solvents were made, and diluted as described later : xylol, benzol, toluol, isobutyl alcohol, amyl alcohol, ethyl alcohol (absolute), ether, acetone, petroleum ether, chloroform. To test the effect of varying concentration upon the position of the fluorescence bands, the fluorescence bands of the xylol solutions with six different concentrations from $\frac{1}{4}$ to $5 \frac{1}{1} 2$ conc. were photographed. There was found to be no apparent shift of the bands with the concentration. The absorption bands of each solution in turn were then photographed, in the following way: The light of an acetylene flame was diffusely reflected from a block of magnesium carbonate at $45^{\circ}$; the solution to be tested was placed, in a glass cell $\mathrm{r} \mathrm{cm}$. in thickness, in the path of the reflected beam, and the spectrograph placed in contact with the cell. All direct light was shut off by a screen. As in other cases, the mercury arc spectrum was used for reference. As an additional precaution against shift with concentration, only dilute solutions were used, the solution in most cases probably being not more than $\frac{1}{250}$ conc. After each exposure, the solution was replaced by the plain solvent in a similar cell, and another exposure made in the same way. Table III. gives the results. The dash indicates that the band extends to the violet end of the visible spectrum.

For the fluorescence bands, the arc light was used. The mercury arc, and sparks of iron, copper, zinc, tin, lead, aluminum, cadmium, antimony, bismuth, silicon, and carbon, failed to give an exciting light sufficiently intense, even when placed directly above the cell at a distance of one inch, and separated from the solution by a quartz plate. In order to guard against the possibility of stray light, the cell was covered with black paper except for two opposite rectangular slits about $\mathrm{I} \mathrm{cm}$. wide, through which the beam of exciting light passed, and for a small circular opening at right angles to these and just fitting the lens of the spectrograph. Also the barrel of the spectrograph was covered with black paper, and 


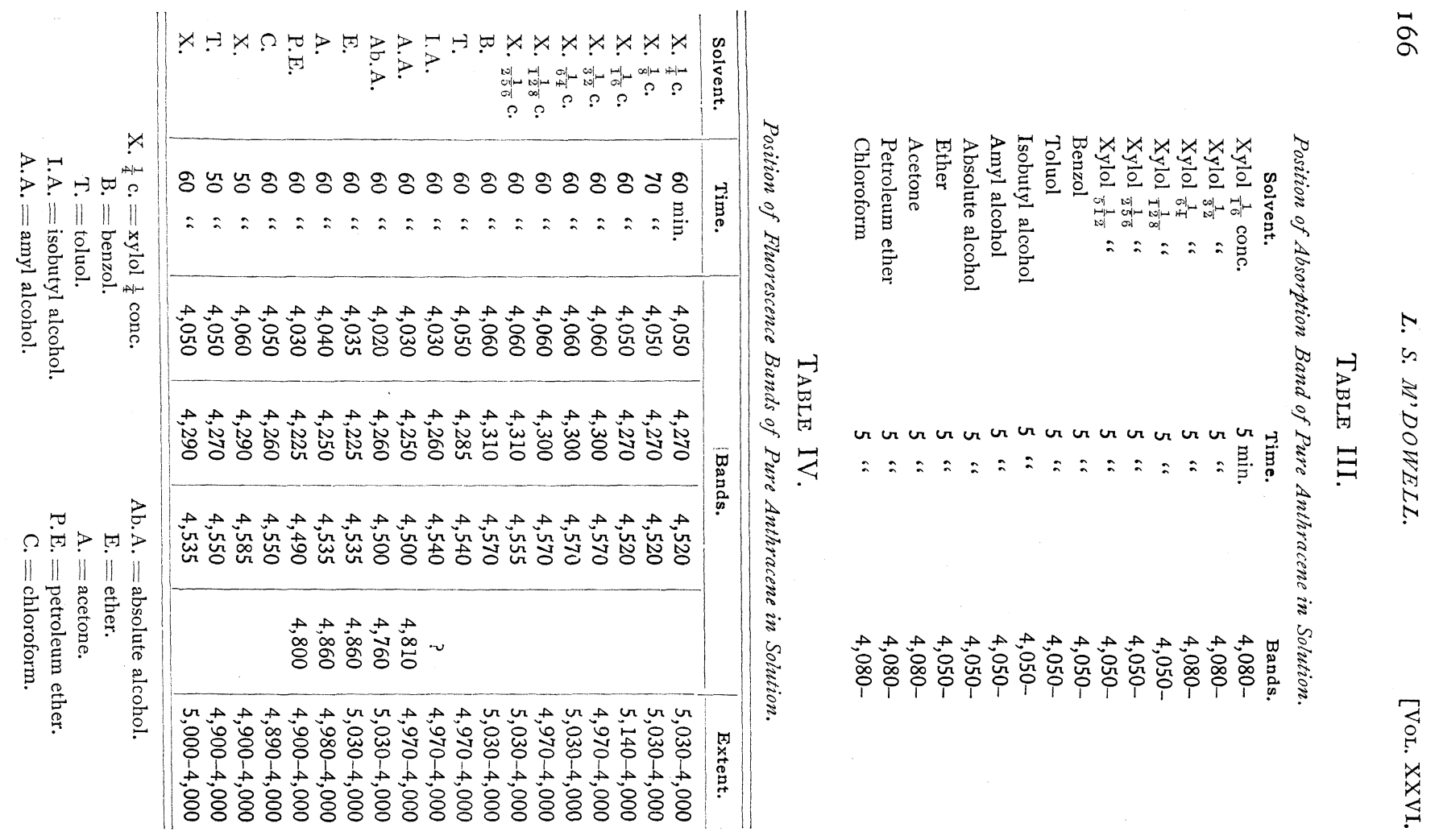


No. 2.] FLUORESCENCE AND ABSORPTION OF ANTHRACENE. I67

had a black screen encircling it at a distance of about two inches from the cell, to prevent any possibility of reflected light entering the instrument.

Of the five exposures made on each plate, the first and third were of the fluorescing solutions, the second and fourth of the plain solvents, the fifth of the mercury arc. As was to be expected, the photographs of the plain solvents were almost blanks, showing the nearly total absence of stray light. In order to determine the exact position of the fluorescence bands, a calibration curve was drawn from the position of the mercury lines. Table IV. gives the results. The midpoint of each band and the total extent of the fluorescence are given in each case. A question mark indicates that the presence of bands in that column is doubtful. For the last exposure the iron arc was used as an exciting light. Otherwise the procedure was the same. See plate.

There was found to be no shift in the position of the bands either with a change in concentration or solvent. Table V. gives the average wave-lengths for the different bands of both pure and impure anthracene in the three forms. The figures for the vapor are those given by T. S. Elston.

\begin{tabular}{|c|c|c|c|c|c|}
\hline \multicolumn{5}{|c|}{ Average Position of Fluorescence Bands. } & \\
\hline Solid. & 4,250 & 4,495 & 4,745 & 4,980 & 5,300 \\
\hline Solution. & 4,050 & 4,275 & 4,540 & 4,820 & \\
\hline Vapor. & 3,900 & 4,150 & 4,320 & & \\
\hline
\end{tabular}

The fluorescence bands occur, apparently, at equal intervals, with a distance between bands approximately equal to $.025 \mu$. With the change from solid to solution and solution to gas, there is a shift in the bands towards the shorter wave-lengths.

\section{Summary.}

The results of the study may be briefly summarized thus :

I. Commercial anthracene, in the solid form, possesses a fluorescence fully as intense as pure anthracene, but different in color. It consists of bright bands at $.498 \mu$ and $.530 \mu$. The absorption spectrum consists of bands in the positions $\lambda .499-.487 \mu, .462-$ $.452 \mu$, and of a continuous spectrum from $\lambda .42$ I $5 \mu$ to some point beyond the range of the apparatus. 
[Vol. XXVI.

II. The blue fluorescence is not entirely destroyed by the presence of the chrysogen, but may with the proper excitation coexist with the green fluorescence.

III. The fluorescence of the pure anthracene, both solid and in solution, consists of distinct bands separated by regions of diminished intensity.

IV. In the case of the solid, there are three bands at $\lambda .425 \mu$, $.450 \mu$, and $.475 \mu$. In the case of the solution, there are three, possibly four, bands at $\lambda .405 \mu, .4275 \mu, .475 \mu$, and (?) $.482 \mu$.

$V$. With the change from solid to solution and solution to gas there is a progressive shift in the fluorescence bands towards the shorter wave-lengths.

VI. The absorption spectrum of the pure anthracene is continuous from about $\lambda .4 \mathrm{I} O \mu$ to some point in the ultra-violet beyond the range of the apparatus. That of the solution is continuous from about $\lambda .405 \mu$.

Physics Laboratory, Cornell University. 
Physicai, Review,

February, I908.

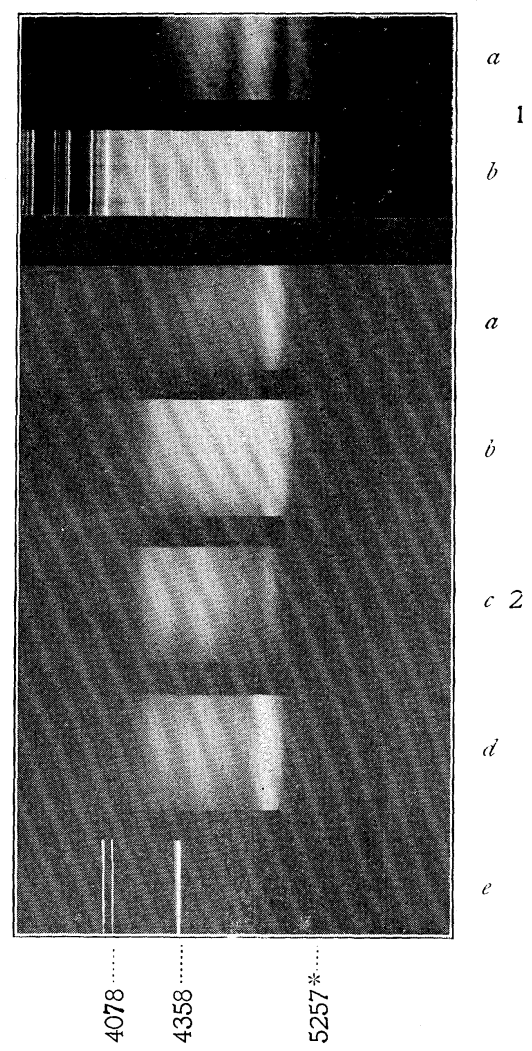

To face page $\mathbf{I} 68$.

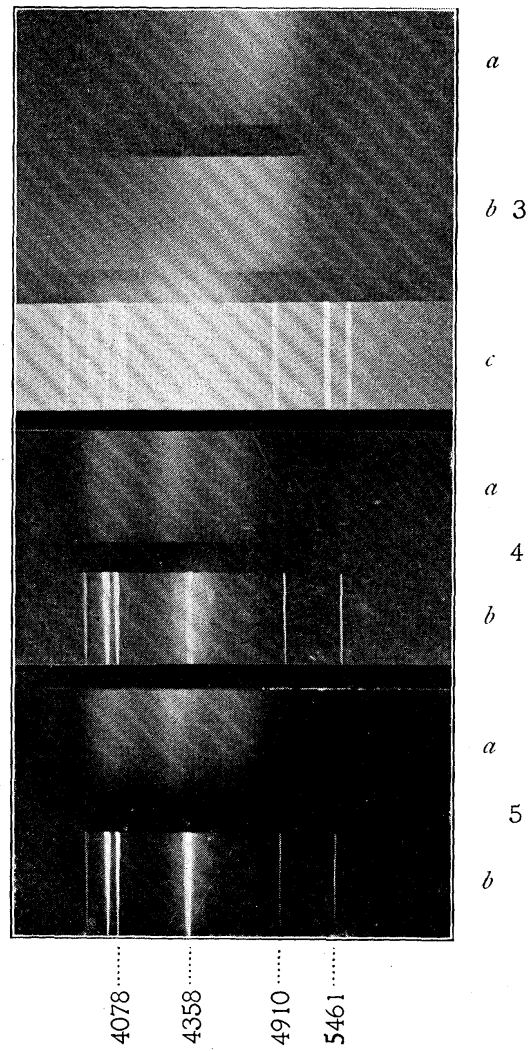

Fluorescence and Absorption Spectra of Anthracene.

I. $a$, Absorption of solid commercial anthracene; $b$, Strontium.

2. Fluorescence of solid anthracene of different degrees of purity (see Table I. ): $a$, Green sublimate; $b$, Commercial anthracene sublimed; $c$, Violet-blue sublimate; $d$, Blue and green sublimate; $e$, Mercury arc.

3. $a$, Absorption of purified anthracene in solution; $b$, Plain solvent ; $c$, Mercury arc.

4. $a$, Fluorescence of purified anthracene in solution, carbon arc excitation ; $b$, Mercury arc.

5. $a$, Fluorescence of purified anthracene in solution, iron arc excitation; $b$, Mercury arc.

$5257^{*}$ is the wave-length of the strontium line at the extreme right. 

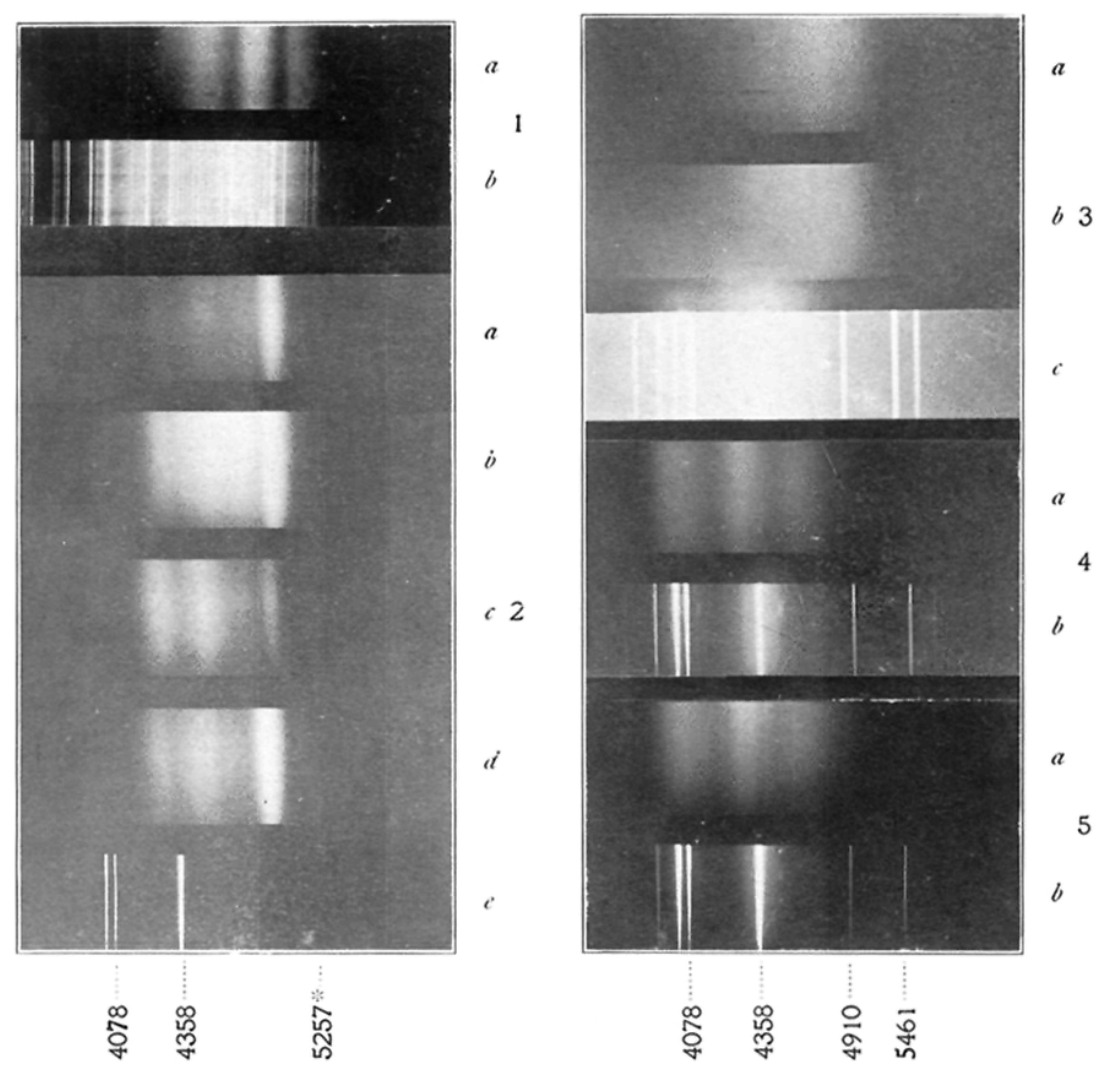

Fluorescence and Absorption Spectra of Anthracene.

I. a, Absorption of solid commercial anthracene; b, Strontium.

2. Fluorescence of solid anthracene of different degrees of purity (see Table I. ): $a$, Green sublimate; $b$, Commercial anthracene sublimed; $c$, Violet-blue sublimate; $d$, Blue and green sublimate; $e$, Mercury arc.

3. $a$, Absorption of purified anthracene in solution; $b$, Plain solvent ; $c$, Mercury arc.

4. a, Fluorescence of purified anthracene in solution, carbon arc excitation ; l, Mercury arc.

5. $a$, Fluorescence of purified anthracene in solution, iron arc excitation; $b$, Mercury arc.

$5257^{*}$ is the wave-length of the strontium line at the extreme right. 